\title{
Multilayerinduktivitäten und Faltflex® als induktives Element in Sensor- und Aktorapplikationen
}

\author{
Anatol Schwersenz, \\ Würth Elektronik GmbH \& Co. KG \\ Salzstrasse 21, 74676 Niedernhall \\ 07940/946-5601, anatol.schwersenz@we-online.de
}

\begin{abstract}
Eine Vielzahl von Sensor- und Aktorfunktionen basieren auf der Wechselwirkung mit elektrischen Feldern. Als Sende- und auch als Empfängerspule werden bis dato fast ausschließlich drahtgewickelte Spulen verwendet. Mit dem Einsatz multilayerbasierende Spulen und der Faltflex-Spulen ergeben sich neue Möglichkeiten.

In Bezug auf die Baugröße können einerseits äußerst kleine Spuleninduktivitäten verwirklicht werden, andererseits aber auch sehr große hochstromfeste Planarinduktivitäten.

Durch definierbare Geometrien im Layout der Platinen können induktive Systeme umgesetzt werden. Mittels Faltflex-Technologie werden Gedankenmodelle mit komplizierten Aufbauten produzierbar. Das Poster zeigt anhand von Anwendungsbeispielen wie die Faltflex-Technologie von Würth Elektronik eingesetzt werden kann.
\end{abstract}

\section{Kurzbiografie}

Anatol Schwersenz, 1986-1991Studium der Physik in Heidelberg, 1991-1995 Studium der physikalischen Technik in Heilbronn, 1995-2000 Prozessingenieur in der Mikrolithografie zur Photomaskenherstellung bei Steag Hamatech, 2000-2004 Produktmanager für Clusterlithografiemaschinen bei Steag-Hamatech, 2004-2007 Leiter der optischen Lithografie am Institut für Mikroelektronik in Stuttgart, 2007-2010 Bereichsleiter Innenlagenfertigung bei Würth Elektronik, ab 2010 Produktmanager für eingebettete Multilayer Induktivitäten (EMI) bei Würth Elektronik.

\section{Multilayer- und Faltflex®-Technologie}

Die Integration induktiver Bauelemente in die Leiterplatte gibt es schon so lange wie die Leiterplatte selbst. Mit jeder neu aufkommenden Fertigungstechnologie wurde auch die Spulentechnik verfeinert. Bis zu dem Stand, dass eine Weiterentwicklung in noch mehr Lagen im Multilayer aus wirtschaftlichen und auflösungsrelevanten Aspekten stagnierte. Die Anforderungen an die Anzahl der Kupferlagen von Planarspulensystemen übersteigen aufgrund ihrer Schaltungskomplexität die für Leiterplattenherstellung üblichen Lagenanzahlen. Daraus resultierend ergeben sich für Spulensysteme nur geringe erreichbare Kupferfüllgrade. Zudem steigt der Platzbedarf bei leiterplattenbasierenden Spulen durch die Vielzahl der zu setzenden Durchkontaktierungen erheblich.

Für die Sensorapplikationen wird auch der störende Einfluss der Durchkontaktierungen im Wechselstromverhalten mit steigender Frequenz immer grösser. Für die meisten sehr kleinen Applikationen ist damit die Fertigung der Spule aus der Standard Leiterplatte nicht möglich.

Mit der Entwicklung von Faltflex wird dem Entwickler eine Technologie an die Hand gegeben, die eine extreme Miniaturisierung zulässt und damit den Zugang zu einer Vielzahl von Sensor- und Aktoranwendungen erschließt. 
In Bezug auf die elektrischen Parameter sind Planarspulen in Faltflex-Technologie vollständig simulierbar und damit optimal für Sensorapplikationen geeignet. Sie weisen durch den geringen Einflusses des Skin-Effekts ein sehr gutes Wechselstromverhalten auf.

Durch die Abbildung der Spulenwirkung in Layoutdaten lassen sich in der Wicklung alle für induktive Systeme interessanten Möglichkeiten abbilden, wie zum Beispiel: Bifilar, Trifilar, Mittelabgriffe, Anzapfungen. Damit werden beispielsweise auch Empfängerspulen in der Sendespule mit dynamischer Vormagnetisierung mit galvanischer Trennung in einem Bauteil möglich. Zudem werden alle weiteren geschachtelten Windungssysteme, wie sie auch für LVDT's (Linear Variable Differential Transformer), RVDT's (Rotational Variable Differential Transformer) und auch richtungsempfindliche Sensoren zur Gestikerkennung benötigt werden, möglich.

In Bezug auf Aktoren sind sehr genau definierbare Wicklungen für zum Beispiel Linearmotoren und andere Auslenkeinrichtungen möglich. Hier können zum einen exzellente Kupferfüllraten erreicht werden, zum anderen kann aber auch wieder eine deutliche Miniaturisierung erreicht werden.

Durch die verwendeten Materialien wird der vielfältigste Einsatz von Faltflex möglich. Hier stehen zum Beispiel die Beständigkeit gegenüber Temperatur, Vakuum, starken Magnetfeldern und auch die chemische Beständigkeit im Vordergrund. Verwendet wird als Basisträgermaterial Polyimid oder LCP (Liquid Cristalline Polymer) und zur elektrischen Leitung Kupfer, für welches sich aufgrund der Fertigungsprozesse annähernd rechteckige Leitungsquerschnitte ergeben.

Mit fortschrittlichsten Fertigungsverfahren werden auf diese Folien beidseitig die Spulen mit den modernsten Leiterplattenfertigungsprozessen produziert. Damit lassen sich Geometrien bis herab zu $25 \mu \mathrm{m}$ Line/Space fertigen. Das entspricht in den Eigenschaften einem 30 $\mu \mathrm{m}$ Draht, dessen Verwendung in Wickeln eine mechanische Herausforderung bei der Herstellung und der Integration in den Sensor stellt.

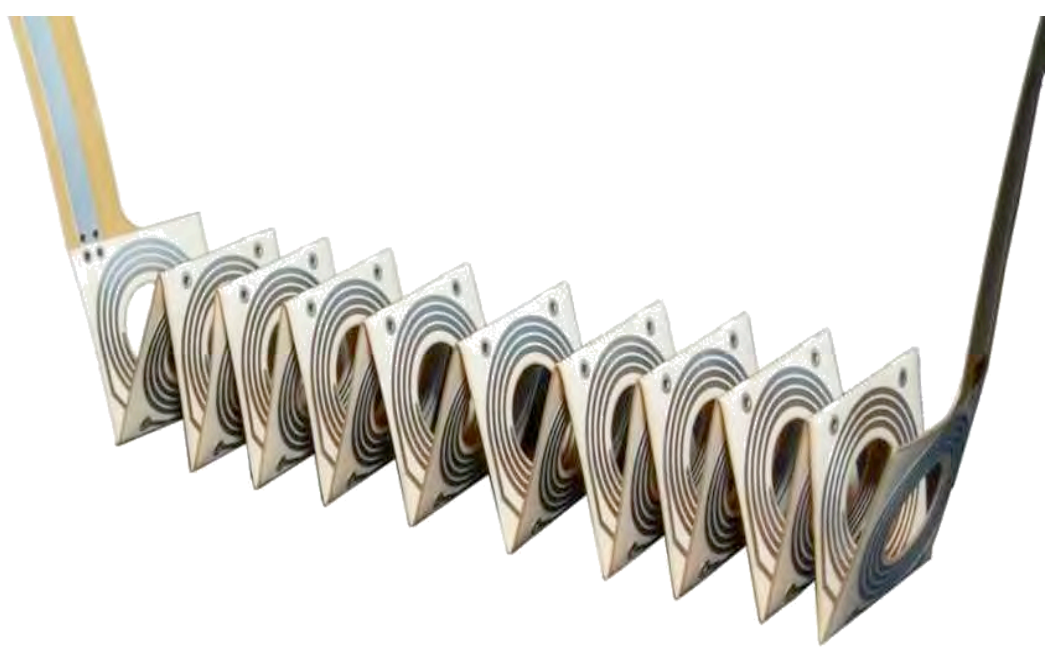

Abbildung 1: Faltflex® auf LCP 


\section{Wirkprinzipien für induktive Sensoren und Aktoren}

Im Wesentlichen basiert der induktive Sensor auf der äußeren Einflussnahme auf das magnetische Feld, dem das Faraday'sche Gesetz zugrunde (rotE=dB/dt) liegt. Damit ergeben sich auch der zeitliche und funktionelle Zusammenhang zwischen der Umwelt und dem Nutzsignal.

Durch geeignete Geometrie des Sensors und dessen Beschaltungen lassen sich die Felder auf die Erkennung von beispielsweise Stoffunterschieden, Richtung, Lage, Wege, Winkel usw. trimmen.

\section{Anwendung von Faltflex ${ }^{\circledR}$ in der Aktor-Sensor Kommunikation}

Mit dem seit letztem Jahr angebotenen eC/DC-Modul für AS-interface Anwendungen gibt es ein standardisiertes Produkt auf dem Markt, welches der symmetrischen Stromauskopplung aus dem Bus dient. Mit diesem Bauteil werden erstmals alle wesentlichen Vorteile der eingebetteten Induktivitäten auf Faltflex-Basis in einem Produkt verwirklicht. Die technischen Parameter sind mit diskreten Bauelementen nicht zu realisieren. Besonders erwähnenswert sind hier die erzielte Symmetrie und die äußerst geringen parasitären Kapazitäten der Induktivitäten.

In der Abbildung 2 ist diese Applikation der Faltflex-Spulen im AS-interface dargestellt, das im Wesentlichen auf die Symmetrieeigenschaften der Koppelspule baut. Ähnlich der Wirkung einer Common-Mode-Choke können hier Gegentaktsignale passieren, während Gleichtaktsignale unterdrückt werden.

Durch die Abbildung der Spule in bildgebenden Daten werden die Parameter sehr wiederholbar und das Verhalten äußerst vorhersehbar. Für die differentiellen Systeme ergeben sich damit eine vereinfachte Signalaufbereitung und auch eine bessere Signalstabilität.

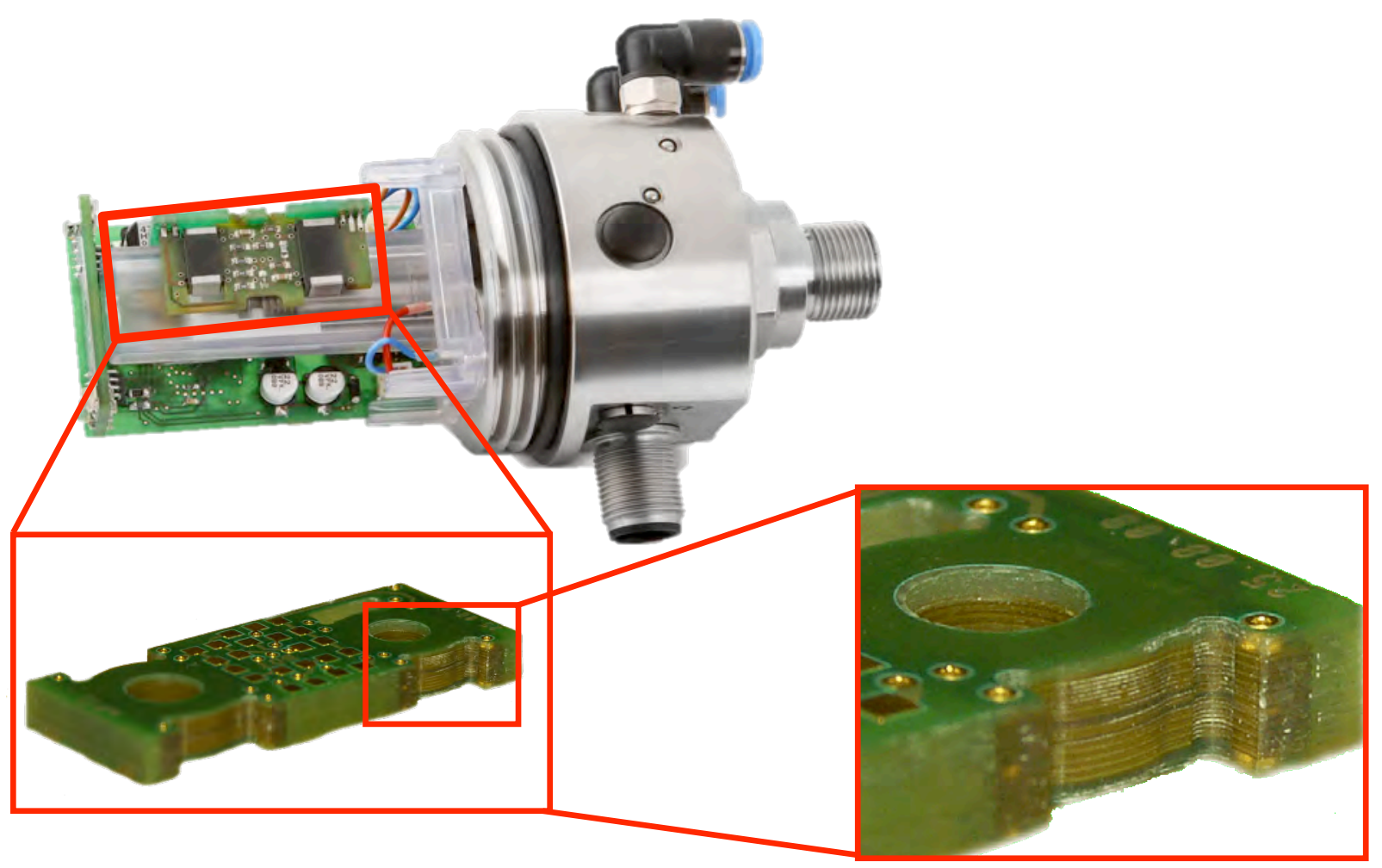


Abbildung 2: Faltflex® als eingettete induktive Komponente in eine Leiterplatte

\section{Geschachtelte Faltflex ${ }^{\circledR}$ in Sensoranwendung}

Von besonderem Interesse wird die Faltflex-Technologie für alle Arten der LVDT (Linear Variable Differential Transformer). Hier wird durch geschicktes Verschalten der einzelnen Spulen ein Messumformer kreiert, mit dem die unterschiedlichsten Messsituationen erfasst werden können. Durch die eindeutige geometrische Zuordnung sind solche Systeme jetzt mit sehr geringen Toleranzen fertigbar.

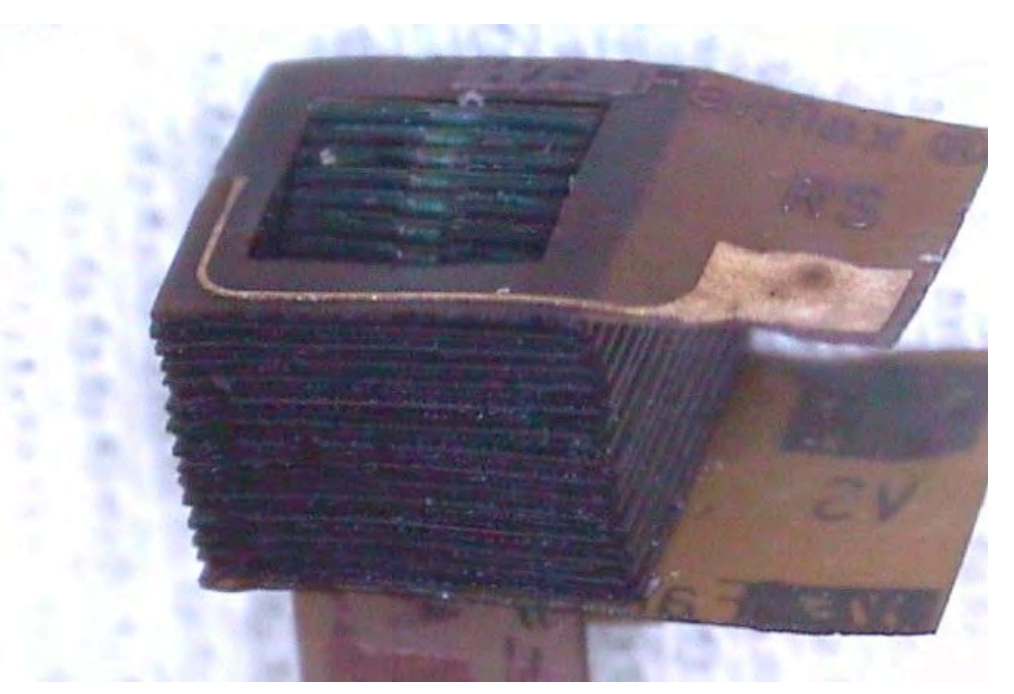

Abbildung 3: Geschachtelter Sensor in Faltflex®-Technologie gefertigt

Der in Abbildung 3 dargestellte Sensor hat eine Kantenlänge von 4,8mm. Die senkrecht dazu angeordnete Innenspule ist 2,9mm x 2,9mm. Der Sensor wird ähnlich einem LVDT betrieben. Da die Felder senkrecht zueinander stehen, gibt es ohne Messobjekt kein Ausgangssignal. Erst mit Auftreten einer äußeren Feldveränderung, die die senkrecht angeordneten Felder ineinander koppelt, entsteht ein Ausgangssignal. Das Ausgangssignal ist damit stark von dem äußeren Einfluss abhängig und somit prädestiniert zum Abtasten von Oberflächeneigenschaften.

\section{Aktorspulen in Faltflex®-Technologie}

Die in Abbildung 4 dargestellten Aktorspulen stellen eine Auswahl von bisher gefertigten Typen dar. Durch das unterschiedlichste Design ist die Faltflex-Technologie an die speziellen Erfordernisse anpassbar. Hier sind sowohl sehr kleine Aktorspulen als auch sehr große hochstromfähige Spulen realisierbar. 


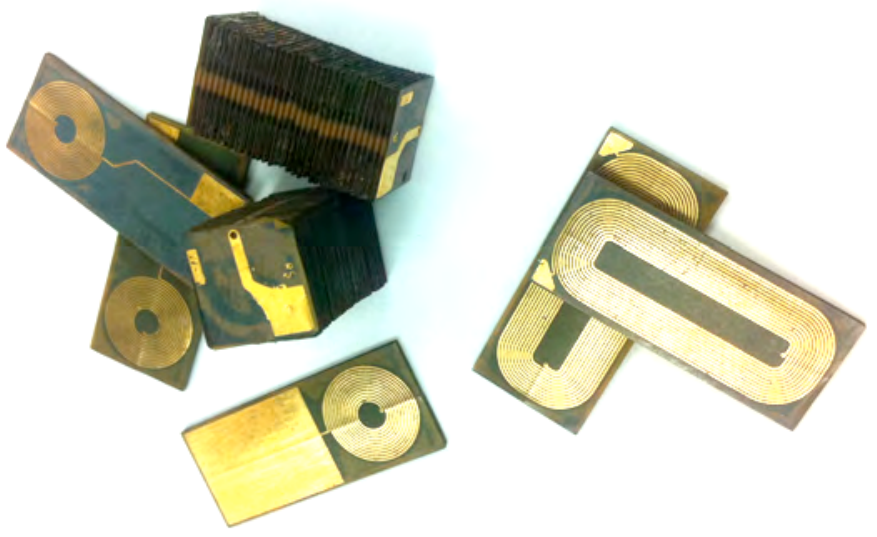

Abbildung 5: Aktorspulen in Faltflex®-Technologie

\section{Zusammenfassung}

Mit der Markteinführung der Faltflex-Technologie in der Anwendung für induktive Systeme ergibt sich für den Anwender eine Vielzahl neuer Möglichkeiten. Sie reicht von der Substitution einer einfachen Induktivität bis hin zu neuartigen Anwendungen, die mit bisherigen Bauelementen nicht verwirklicht werden konnten.

Beispiel hierfür sind das eC/DC-Modul und der geschachtelte induktive Sensor. Diese Beispiele setzen sich mit ihren technischen Parametern deutlich von den machbaren drahtbasierenden Lösungen ab. Dem Anwender bietet sich mit Faltflex eine neue Möglichkeit, mit der in einem sehr hohen Freiheitsgrad induktive Bauelemente designet werden können. Die Anwendung und somit die Geometrie und die Verschaltung der Spulensegmente treten hierbei in den Vordergrund.

Aufgrund der fortschreitenden Miniaturisierung in den Fertigungsprozessen werden in naher Zukunft Faltflex-Induktivitäten auch im Chip-Format realisierbar sein. 